\title{
One-loop quantum gravity repulsion in the early Universe*
}

\author{
Bogusław Brodat \\ Department of Theoretical Physics, University of Eódź, \\ Pomorska 149/153, PL-92-002 Eódź, Poland
}

\begin{abstract}
Perturbative quantum gravity formalism is applied to compute the lowest order corrections to the classical spatially flat cosmological FLRW solution (for the radiation). The presented approach is analogous to the approach applied to compute quantum corrections to the Coulomb potential in electrodynamics, or rather to the approach applied to compute quantum corrections to the Schwarzschild solution in gravity. In the framework of the standard perturbative quantum gravity, it is shown that the corrections to the classical deceleration, coming from the one-loop graviton vacuum polarization (self-energy), have (UV cutoff free) opposite to the classical repulsive properties which are not negligible in the very early Universe. The repulsive "quantum forces" resemble those known from loop quantum cosmology.

Keywords: one-loop graviton vacuum polarization; one-loop graviton self-energy; quantum corrections to classical gravitational fields; early Universe; quantum cosmology.

PACS (2010) numbers: 04.60.Gw Covariant and sum-over-histories quantization; 98.80.Es Observational cosmology (including Hubble constant, distance scale, cosmological constant, early Universe, etc): 04.60.Bc Phenomenology of quantum gravity; 04.60.Pp Loop quantum gravity, quantum geometry, spin foams.
\end{abstract}

*Dedicated to Jakub Rembieliński on the occasion of his 65 th birthday.

${ }^{\dagger}$ Electronic address: bobroda@uni.lodz.pl URL: http://merlin.fic.uni.lodz.pl/kft/people/BBroda 
Introduction. The aim of our work it to explicitly show the appearance of "repulsive forces" of quantum origin in the very early Universe. As fundamental guiding references to our letter we would like to point out the publications devoted to perturbative calculations of quantum corrections to classical electromagnetic (the Uehling potential) and gravitational fields. More precisely, we would mean, for example, the works presenting one-loop quantum corrections to the Coulomb potential in electrodynamics (see, e.g. $\S 114$ in [1]), or rather the lowest order quantum corrections to the Schwarzschild solution in gravity (see, [2], and also, e.g. [3]). Actually, we apply the method successfully used in the case of the Schwarzschild solution in [2] to the spatially flat Friedmann-Lemaitre-Robertson-Walker (FLRW) solution (for the radiation). Fortunately, it appears that the cosmological FLRW case is only a little bit more complicated than Schwarzschild's one, and moreover our results conform with the present knowledge. Namely, the lowest order quantum corrections coming from the fluctuating graviton vacuum yield "repulsive forces" resembling the well-known situation in loop quantum cosmology (LQC). The phenomenon is negligible in our epoch, but it is not so in the very early Universe. Moreover, it appears, the result is UV cutoff-free, despite the fact that the cutoff has been primarily imposed (see, e.g. [3]). One should stress that our derivation is a lowest order approximation - the graviton vacuum polarization (self-energy) is taken in one-loop approximation, and the approach assumes the validity of the weak-field regime.

Quantum corrections. Our starting point is a general spatially flat FLRW metric

$$
d s^{2}=g_{\mu \nu} d x^{\mu} d x^{\nu}=d t^{2}-a^{2}(t) \boldsymbol{d} \boldsymbol{r}^{2}
$$

with the cosmic scale factor $a(t)$. To satisfy the condition of the weakness of the (perturbative) gravitational field $\kappa h_{\mu \nu}$ near our reference time $t=t_{0}$ in the expansion

$$
g_{\mu \nu}=\eta_{\mu \nu}+\kappa h_{\mu \nu}
$$

( $\kappa=\sqrt{32 \pi G_{N}}$, with $G_{N}$ - the Newton gravitational constant), the metric is rescaled such a way that it is exactly Minkowskian for $t=t_{0}$, i.e.

$$
a^{2}(t)=1-\kappa h(t), \quad h\left(t_{0}\right)=0 .
$$

Then

$$
h_{\mu \nu}(t, \boldsymbol{r})=h(t) \mathcal{I}_{\mu \nu} \quad \text { and } \quad \mathcal{I}_{\mu \nu} \equiv\left(\begin{array}{cc}
0 & 0 \\
0 & \delta_{i j}
\end{array}\right)
$$


In view of the standard harmonic gauge condition (see, the second eq. in (8) ) which is to be imposed in a moment, we perform the following gauge transformation:

$$
\kappa h_{\mu \nu} \rightarrow \kappa h_{\mu \nu}^{\prime}=\kappa h_{\mu \nu}+\partial_{\mu} \xi_{\nu}+\partial_{\nu} \xi_{\mu} \quad \text { with } \quad \xi_{\mu}(t)=\left(-\frac{3 \kappa}{2} \int_{0}^{t} h\left(t^{\prime}\right) d t^{\prime}, 0,0,0\right) .
$$

Skipping the prime for simplicity, we get

$$
h_{\mu \nu}(t, \boldsymbol{r})=h(t)\left(\begin{array}{cc}
-3 & 0 \\
0 & \delta_{i j}
\end{array}\right) \quad \text { and } \quad h_{\lambda}^{\lambda}(t)=-6 h(t)
$$

where indices are being manipulated with the Minkowski metric $\eta_{\mu \nu}$. Switching from $h_{\mu \nu}$ to standard ("better") perturbative gravitational variables, namely to the "barred" field $\bar{h}_{\mu \nu}$ defined by

$$
\bar{h}_{\mu \nu} \equiv h_{\mu \nu}-\frac{1}{2} \eta_{\mu \nu} h_{\lambda}^{\lambda}
$$

we get

$$
\bar{h}_{\mu \nu}(t, \boldsymbol{r})=-2 h(t) \mathcal{I}_{\mu \nu} \quad \text { with } \quad \partial^{\mu} \bar{h}_{\mu \nu}=0
$$

The Fourier transform of $\bar{h}_{\mu \nu}$ is given by

$$
\tilde{\bar{h}}_{\mu \nu}(p)=-2 \tilde{h}(E)(2 \pi)^{3} \delta^{3}(\boldsymbol{p}) \mathcal{I}_{\mu \nu}
$$

To obtain quantum corrections to a classical field (line) we should supplement the classical line with a vacuum polarization (self-energy) contribution and a corresponding (full) propagator. Therefore, the lowest order quantum corrections $\tilde{\bar{h}}_{\mu \nu}^{q}$ to the classical gravitational field $\tilde{\overline{h_{\mu \nu}^{c}}}$ are given, in the momentum representation, by the formula (see, e.g. [2], or $\S 114$ in [1] for an electrodynamic version - the Uehling potential)

$$
\tilde{\overline{h_{\mu \nu}^{\mathrm{q}}}}(p)=\left(D \Pi \tilde{\overline{\bar{h}}^{\mathrm{c}}}\right)_{\mu \nu}(p)
$$

where

$$
D_{\mu \nu}^{\alpha \beta}(p)=\frac{i}{p^{2}} \mathbb{D}_{\mu \nu}^{\alpha \beta}
$$

is the free graviton propagator in the harmonic gauge with the auxiliary (constant) tensor $\mathbb{D}$ defined in Eq.(12) below, and $\Pi_{\mu \nu}^{\alpha \beta}(p)$ is the (one-loop) graviton vacuum polarization (self-energy) tensor operator. Now, we are defining the following useful auxiliary tensors:

$$
\mathbb{D} \equiv \mathbb{E}-2 \mathbb{P}, \quad \text { where } \quad \mathbb{E}_{\mu \nu}^{\alpha \beta} \equiv \frac{1}{2}\left(\delta_{\mu}^{\alpha} \delta_{\nu}^{\beta}+\delta_{\nu}^{\alpha} \delta_{\mu}^{\beta}\right) \quad \text { and } \quad \mathbb{P}_{\mu \nu}^{\alpha \beta} \equiv \frac{1}{4} \eta^{\alpha \beta} \eta_{\mu \nu}
$$


which satisfy the following obvious identities:

$$
\mathbb{E}^{2}=\mathbb{E}, \quad \mathbb{P}^{2}=\mathbb{P}, \quad \mathbb{E} \mathbb{P}=\mathbb{P E}=\mathbb{P} \quad \text { and } \quad \mathbb{D}^{2}=\mathbb{E}
$$

By virtue of the definition (7), we observe that

$$
\bar{h}_{\mu \nu}=(\mathbb{D} h)_{\mu \nu} .
$$

Multiplying Eq.(10) from the left by $\mathbb{D}$, we obtain (using (11), (14), and the last identity in the series (13) )

$$
\tilde{h}_{\mu \nu}(p)=\frac{i}{p^{2}}\left(\Pi \tilde{\overline{h^{\mathrm{c}}}}\right)_{\mu \nu}(p)
$$

Actually, a useful simplification takes place in (15), namely,

$$
\tilde{h}_{\mu \nu}^{\mathrm{q}}(p)=\frac{i}{p^{2}}\left(\Pi^{\prime} \tilde{\bar{h}}^{\mathrm{c}}\right)_{\mu \nu}(p)
$$

where $\Pi^{\prime}(p)$ is an "essential" part of the full (in one-loop approximation) graviton polarization operator $\Pi(p)$. The "essential" part $\Pi^{\prime}(p)$ of the full (one-loop) graviton vacuum polarization operator $\Pi(p)$ is obtained from $\Pi(p)$ by skipping all the terms with the momenta $p$ with free indices (e.g. $\alpha, \beta, \mu$, or $\nu$ ). Such a simplification follows from the gauge freedom the $\tilde{h}_{\mu \nu}$ enjoys, and from the harmonic gauge condition the $\tilde{\bar{h}}_{\alpha \beta}^{\mathrm{c}}$ satisfies. In general, by virtue of symmetry of indices, $\Pi(p)$ consists of 5 (tensor) terms. Each $p_{\mu}$ can be ignored in $\Pi(p)$ because it only generates a gauge transformation of $\tilde{h}_{\mu \nu}$. Moreover, since $\overline{\tilde{h}}_{\alpha \beta}$ satisfies the harmonic gauge condition, the terms with $p^{\alpha}$ in $\Pi(p)$ are being annihilated. In other words,

$$
\Pi(p)=\underbrace{\Pi^{\prime}(p)}_{2 \text { terms }}+\underbrace{\cdots p}_{3 \text { skipped terms }} .
$$

Since the momenta $p$ in the ellipses posses free indices, they can be ignored, and only the first two terms with dummy indices $\left(p^{2}\right)$ survive $\left(\Pi^{\prime}(p)\right)$. Thus,

$$
\Pi^{\prime}(p)=\kappa^{2} p^{4} I\left(p^{2}\right)\left(2 \alpha_{1} \mathbb{E}+4 \alpha_{2} \mathbb{P}\right)
$$

where the numerical values of the coefficients $\alpha_{1}$ and $\alpha_{2}$ depend on the kind of the virtual field circulating in the loop, and the (scalar) standard loop integral $I\left(p^{2}\right)$ with the UV cutoff $M$ is asymptotically of the form (see, e.g., Chapt. 9.4.2 in [4])

$$
I\left(p^{2}\right)=\frac{1}{(2 \pi)^{4}} \int_{\text {UV cutoff }=M} \frac{d^{4} q}{q^{2}(p-q)^{2}}=-\frac{i}{(4 \pi)^{2}} \log \left(-\frac{p^{2}}{M^{2}}\right)+\cdots
$$


where the dots mean terms $\mathcal{O}\left(p^{2} / M^{2}\right)$. A standard way to derive (19) consists in continuing $q_{0}$ to $+i q_{4}$ (which corresponds to Euclidean formalism, $d^{4} q \rightarrow i d^{4} q_{\mathrm{E}}$ ), exponentiating the denominator using a (double) proper-time representation for the propagators, a change of proper-time variables, imposing the UV cutoff for a new proper time, and continuing back to the Minkowskian momentum. Thus, we obtain

$$
\begin{aligned}
\tilde{h}_{\mu \nu}^{\mathrm{q}}(p) & =\frac{i}{p^{2}} \kappa^{2} p^{4}\left[-\frac{i}{(4 \pi)^{2}} \log \left(-\frac{p^{2}}{M^{2}}\right)\right]\left[-2 \tilde{h}^{\mathrm{c}}(E)(2 \pi)^{3} \delta^{3}(\boldsymbol{p})\right]\left[\left(2 \alpha_{1} \mathbb{E}+4 \alpha_{2} \mathbb{P}\right) \mathcal{I}\right]_{\mu \nu} \\
& =-2 \pi \kappa^{2} E^{2} \log \left|\frac{E}{M}\right| \tilde{h^{\mathrm{c}}}(E) \delta^{3}(\boldsymbol{p})\left(\begin{array}{cc}
-3 \alpha_{2} & 0 \\
0 & \left(2 \alpha_{1}+3 \alpha_{2}\right) \delta_{i j}
\end{array}\right) .
\end{aligned}
$$

The unnecessary modulus sign in Eq.(20) is only to remind the fact that there is also an imaginary contribution to the metric due to creation processes which are ignored in our further analysis.

Radiation source. Now, we should specify our input classical metric. For definiteness, we choose the radiation as a source (the early Universe), but it is not crucial, and assume

$$
a^{2}(t)=\theta(t)\left(\frac{t}{t_{0}}\right)
$$

Then

$$
\kappa h^{\mathrm{c}}\left(t_{0}\right)=0, \quad \kappa \dot{h^{\mathrm{c}}}\left(t_{0}\right)=-\frac{1}{t_{0}} \quad \text { and } \quad \kappa \ddot{h^{\mathrm{c}}}\left(t_{0}\right)=0 .
$$

By virtue of the definition of the deceleration parameter $q$, expressed by

$$
q\left(t_{0}\right) \equiv-\frac{a \ddot{a}}{(\dot{a})^{2}}\left(t_{0}\right)=1+2\left[1-\kappa h\left(t_{0}\right)\right] \frac{\kappa \ddot{h}\left(t_{0}\right)}{\left(\kappa \dot{h}\left(t_{0}\right)\right)^{2}},
$$

we immediately get the classical result

$$
q^{\mathrm{c}}\left(t_{0}\right)=1
$$

According to (3) and (21) the Fourier transform of $h^{\mathrm{c}}(t)$ is

$$
\tilde{h^{\mathrm{c}}}(E)=\frac{1}{\kappa t_{0}}\left(\frac{1}{E^{2}}+\cdots\right)
$$

where the dots mean terms (vanishing in the next formula) proportional to the Dirac delta and its first derivative. Hence

$$
\tilde{h}^{\mathrm{q}}{ }_{\mu \nu}(p)=-\frac{2 \pi \alpha \kappa}{t_{0}} \log \left|\frac{E}{M}\right| \delta^{3}(\boldsymbol{p}) \mathcal{I}_{\mu \nu},
$$




\begin{tabular}{|c|c|c|c|}
\hline spin & $\alpha_{1}$ & $\alpha_{2}$ & $\alpha$ \\
\hline \hline 0 & $\frac{1}{480}$ & $-\frac{1}{720}$ & 0 \\
\hline$\frac{1}{2}$ & $\frac{1}{160}$ & $-\frac{1}{240}$ & 0 \\
\hline 1 & $\frac{1}{40}$ & $-\frac{1}{60}$ & 0 \\
\hline 2 & $\frac{27}{80}$ & $-\frac{59}{240}$ & $-\frac{1}{16}$ \\
\hline
\end{tabular}

Table I: Coefficients $\alpha_{1}$ and $\alpha_{2}$ entering the one-loop graviton vacuum polarization (self-energy) tensor operator (18) (taken from [5] 9$]$ ); $\alpha \equiv 2 \alpha_{1}+3 \alpha_{2}$.

where $\alpha \equiv 2 \alpha_{1}+3 \alpha_{2}$, and performing the gauge transformation in the spirit of (5), we have removed the purely time component of $h^{\mathrm{q}}{ }_{\mu \nu}$, i.e. $h^{\mathrm{q}}{ }_{00} \rightarrow h^{\mathrm{q}^{\prime}}=0$. The inverse Fourier transform yields

$$
h_{\mu \nu}^{\mathrm{q}}(t)=\frac{2 \pi^{2} \alpha \kappa}{(2 \pi)^{4} t_{0}}\left(|t|^{-1}+\cdots\right) \mathcal{I}_{\mu \nu}
$$

where this time the dots mean a term (vanishing for $t>0$ ) proportional to the Dirac delta. Therefore, for $t>0$ we have

$$
\kappa h^{\mathrm{q}}(t)=\frac{\alpha \kappa^{2}}{8 \pi^{2} t_{0}} t^{-1}=-\frac{G}{4 \pi t_{0}} t^{-1}
$$

where according to Table【 only the graviton field contributes with $\alpha=-\frac{1}{16}$. Now,

$$
\kappa h^{\mathrm{q}}\left(t_{0}\right)=-\frac{G}{4 \pi t_{0}^{2}}, \quad \kappa \dot{h^{\mathrm{q}}}\left(t_{0}\right)=\frac{G}{4 \pi t_{0}^{3}} \quad \text { and } \quad \kappa \ddot{h^{\mathrm{q}}}\left(t_{0}\right)=-\frac{G}{2 \pi t_{0}^{4}} .
$$

The total graviton field $\kappa h=\kappa h^{\mathrm{c}}+\kappa h^{\mathrm{q}}$, and its derivatives at the time $t_{0}$, expressed in the dimensionless (Planck's) time unit

$$
\tau \equiv \frac{1}{\sqrt{G}} t_{0}
$$

are

$$
\kappa h(\tau)=-\frac{1}{4 \pi \tau^{2}}, \quad \kappa \dot{h}(\tau)=-\frac{1}{t_{0}}\left(1-\frac{1}{4 \pi \tau^{2}}\right) \quad \text { and } \quad \kappa \ddot{h}(\tau)=-\frac{1}{t_{0}^{2}}\left(\frac{1}{2 \pi \tau^{2}}\right) .
$$

Finally, by virtue of (23), we obtain the total deceleration parameter of the form

$$
q(\tau)=1-\frac{1}{\pi \tau^{2}}+\mathcal{O}\left(\tau^{-4}\right)
$$


Final remarks. In the framework of the standard (one-loop) perturbative quantum gravity, we have derived the formula (32) expressing the value of the total (effective) deceleration parameter $q(\tau)$. The quantum contribution, $\delta q(\tau)=q(\tau)-q^{\mathrm{c}}(\tau) \approx-\frac{1}{\pi \tau^{2}}$, is negligible in our epoch, but certainly it could play a role in a very early evolution of the Universe. Perturbative nature of the approach imposes bounds on the applicability of the result, but nevertheless one can observe its distinctive features: actually, it is an independent perturbative confirmation of the existence of strong repulsive (singularity resolving) forces typically being attributed to the realm of LQC (cosmological bounce); inputs and outputs are consequently given in terms of the metric tensor; only pure gravity contributes to our result (see $\alpha$ in Table I); and finally, no trace of the UV cutoff is present anywhere.

Nowadays, loop quantum gravity (LQG) or, in the context of cosmology, LQC is the most promising approach towards quantization of gravity and proper treatment of the very early evolution of the Universe. It is interesting to compare our repulsion and the LQC bounce. In LQC, the effective Friedmann equation is modified for extremely high densities of the order of the so-called critical density $\rho_{\text {crit }}$. The modification amounts to the appearance of strong repulsion (bounce) for the densities of the order of $\rho_{\text {crit }}$ which are expected in the very early Universe. The LQC bounce resembles our repulsion. But there is some important difference between the both approaches, besides the obvious technical differences. Namely, in LQC the effect is due to matter fields (the density of matter $\rho$ ), whereas in our approach the whole effect is exclusively due to graviton contribution to graviton vacuum polarization (self-energy). In fact, according to the Table I no matter field contributes to the repulsion.

Supported by the University of Łódź grant.

[1] V. B. Berestetskii, L. P. Pitaevskii, and E. M. Lifshitz, Quantum Electrodynamics, vol. 4 (Butterworth-Heinemann, 1982), 2nd ed.

[2] M. J. Duff, Phys. Rev. D 9, 1837 (1974).

[3] J. F. Donoghue, Phys. Rev. Lett. 72, 2996 (1994).

[4] K. Huang, Quarks, Leptons and Gauge Fields (World Scientific Publishing Company, 1992), 2nd ed.

[5] D. M. Capper and M. J. Duff, Nucl. Phys. B82, 147 (1974). 
[6] D. M. Capper, G. Leibbrandt, and M. R. Medrano, Phys. Rev. D 8, 4320 (1973).

[7] M. J. Duff and J. T. Liu, Phys. Rev. Lett. 85, 2052 (2000).

[8] D. Capper, Il Nuovo Cim. 25A, 29 (1975).

[9] D. M. Capper, M. J. Duff, and L. Halpern, Phys. Rev. D 10, 461 (1974). 